\title{
FRACTIONAL BROWNIAN POLYMERS - SOME FIRST RESULTS
}

\author{
JINKY BORNALES \\ Physics Department, MSU-IIT, Iligan City, The Philippines \\ jinky.bornales@g.msuiit.edu.ph \\ SAMUEL ELEUTÉRIO \\ CFTP - Physics Department, Instituto Superior Técnico, \\ Universidade Técnica de Lisboa, Portugal \\ sme@ist.utl.pt \\ MARIA JOÃO OLIVEIRA \\ Univ. Aberta, Portugal \\ CMAF, Univ. Lisbon, Portugal \\ oliveira@cii.fc.ul.pt \\ LUDWIG STREIT \\ CCM, Univ. Madeira, Portugal \\ BiBoS, Univ. Bielefeld, Germany \\ streit@physik.uni-bielefeld.de
}

\begin{abstract}
Recently the Edwards model for chain polymers in good solvents has been extended to include fractional Brownian motion trajectories as a description of polymer conformations. This raises in particular the question of the corresponding Flory formula for the end-to-end length of those molecules. A generalized Flory formula has been proposed, and there are some first results of numerical validations.
\end{abstract}

Keywords: Fractional Brownian motion. Flory index. Edwards model. Polymers.

PACS numbers: 11.25.Hf, $123.1 \mathrm{~K}$

\section{The Edwards Model for Chain Polymers in Solvents}

Brownian motion paths $x(t), 0 \leq t \leq N$, provide a useful model for the conformation of chain polymers in good solvents and have been extensively studied in $d=1,2$, and 3 dimensions. Their self-crossings, however, are uncharacteristic for such conformations and should be suppressed ("excluded volume effect"). In the Edwards model $^{6}$ this is done using the self-intersection local time

$$
L=\iint d s d t \delta(x(s)-x(t))
$$


of Brownian motion; formally we modify the Wiener measure $d \mu(x)$ by a density function $Z^{-1} \exp (-g L), g>0$, where the normalization

$$
Z=\mathbb{E}\left(\exp \left(-g \int_{0}^{N} d s \int_{0}^{N} d t \delta(x(s)-x(t))\right)\right)=\int \exp (-g L) d \mu
$$

should be chosen so that $Z^{-1} \exp (-g L) d \mu$ is a probability measure. Mathematically this is tricky: The above expressions can be made well-defined, ${ }^{9}$ e.g. as limit of suitably regularized versions with

$$
Z=\lim _{\varepsilon} \int \exp \left(-g L_{\varepsilon}\right) d \mu
$$

in the unphysical case of $d=1$. This is not so in higher dimensions. For $d=2$ the expectation of the local time is divergent, and we need to "center" the local time by subtracting its expectation, i.e. we need to replace

$$
L_{\varepsilon} \rightarrow L_{\varepsilon}-\mathbb{E}\left(L_{\varepsilon}\right)
$$

before taking the limit. In three dimensions an additional multiplicative renormalization is required.

\section{Fractional Brownian Motion Models}

A more general class of models is obtained if we model the conformations $x(t)$ by fractional Brownian motion (fBm) paths. Fractional Brownian motion with "Hurst parameter" $H \in(0,1)$ can be characterized ${ }^{2,12}$ as a centered Gaussian process with covariance

$$
\mathbb{E}\left(B_{i}^{H}(s) B_{j}^{H}(t)\right)=\frac{\delta_{i j}}{2}\left(t^{2 H}+s^{2 H}-|t-s|^{2 H}\right), \quad i, j=1, \ldots, d, s, t \geq 0 .
$$

(We see that $H=1 / 2$ is standard Brownian motion.)

More realistically, instead of just using $\mathrm{fBm}$, the excluded volume should again be introduced as above. Of course then the question of mathematical existence arises again, and a (partial) answer has recently been given in: ${ }^{8}$

\section{Theorem 2.1.}

The Edwards model is well defined for $x=B^{H}$ whenever $H \leq 1 / d$.

It is important to note that $\mathrm{fBm}$ is a process with stationary increments, in keeping with chain polymer homogeneity, contrary to models using e.g.

$$
x(t)=B\left(t^{H}\right) .
$$

On the other hand, $\mathrm{fBm}$ is not Markovian, a fact which complicates considerably the mathematical analysis of the corresponding polymer models. 


\subsection{The interaction between monomers}

Usually, infinite dimensional path integrals are defined via finite dimensional approximations, but in the present context the situation is the opposite: the path integral model is an idealization of the finite dimensional integration over all monomer positions $x_{k}$. For $\mathrm{N}$ monomers we set

$$
\begin{gathered}
x_{k}=x(k)=B^{H}(k), \quad k=0, \ldots, N-1 \\
\int d \mu(x) \approx \text { const. } \prod_{k=0}^{N-1} \int d x_{k} \exp \left(-H_{0}(x)\right) .
\end{gathered}
$$

It is easy to determine the quadratic form $H_{0}(x) \equiv \frac{1}{2}\left(x, h_{0} x\right)$, since for any positive matrix $h_{0}$ we have

$$
\begin{aligned}
\mathbb{E}(\exp (x, b)) & =\left((2 \pi)^{n} \operatorname{det} h_{0}\right)^{-1 / 2} \int d^{n} x \exp \left(-\frac{1}{2}\left(x, h_{0} x\right)+(b, x)\right) \\
& =\exp \left(\frac{\left(b, h_{0}^{-1} b\right)}{2}\right) .
\end{aligned}
$$

Computing

$$
\left|\frac{\partial}{\partial b_{k}} \frac{\partial}{\partial b_{l}} \mathbb{E}(\exp (x, b))\right|_{b=0}=\mathbb{E}\left(x_{k} x_{l}\right)=\left(h_{0}^{-1}\right)_{k l}
$$

we see that the matrix $h_{0}$ is just the inverse of the covariance function given by (1):

$$
\left(h_{0}^{-1}\right)_{k l}=\mathbb{E}\left(B^{H}(k) B^{H}(l)\right) .
$$

For the standard Brownian motion the well-known result is

$$
H_{0}(x)=\frac{1}{2} \sum\left(x_{k}-x_{k-1}\right)^{2}
$$

i.e. an attractive quadratic interaction between nearest neighbors. For $N=9$ we display the matrix elements

$$
h_{4, k}=\left\{\begin{array}{c}
2 \text { if } \quad k=5 \\
-1 \text { if } \quad k=4,6 \\
0 \quad \text { otherwise }
\end{array} .\right.
$$

in Figure 1.

In the general case, a numerical inversion of the covariance matrix (2) can be done. For small $H$ we find long range attraction corresponding to curlier polymers (Fig. 2), while for bigger $H$ we observe a next-to-nearest neighbor repulsion (Fig. 3) which straightens them out. 


\section{The End-to-End Length}

Central to the study of polymer conformations is the question of how their end-toend distance $R$, with

$$
R^{2}=\mathbb{E}\left(\left|x_{N-1}\right|^{2}\right)
$$

in our notation, scales with $N$ as the number of monomers becomes large. In the ansatz

$$
R \sim N^{v}
$$

$v$ is the famous "Flory index" which was originally conjectured to depend on the dimension $d$ as follows:

$$
v=\frac{3}{d+2}
$$

Theoretical studies support this conjecture for $d=1,2$ while various less rigorous methods, from computer simulations to renormalization group theory,point to a slight deviation for $d=3$, in agreement with experimental results:

$$
\begin{aligned}
& \text { Flory Theory Exp. } \\
& d=1: \quad 1 \quad 1 \\
& d=2: 0.75 \quad 0.75 \quad 0.79 \\
& d=3: 0.60 \quad 0.588 \quad 0.59 \\
& d=4: \quad 0.5 \quad 0.5
\end{aligned}
$$

\subsection{Extension to the fractional case}

Recently Bornales, Oliveira, and Streit ${ }^{5}$ have proposed a generalization of Flory's conjecture to the fractional case with general Hurst index $H$ :

$$
v_{H}=\left\{\begin{array}{ccc}
1 & \text { if } d=1 \text { and } H>1 / 2 \\
H & \text { if } & d H>2 \\
\frac{2 H+2}{d+2} & \text { if } & \text { otherwise. }
\end{array} .\right.
$$

Here the first condition stems from the fact that the scaling exponent $v=1$ is maximal, polymers will not grow faster than the number of monomers. The second condition reflects the fact that for $d H=2 \mathrm{fBm}$ becomes self-intersection free, ${ }^{14}$ beyond that value the end-to-end-length will scale like for free $\mathrm{fBm}$.

We mention in passing that there is also a proposition for a recursion formula, generalizing the one given by Kosmas and Freed ${ }^{11}$ for the case of conventional Brownian motion. 


\subsection{Computer simulations - first results}

As mentioned above, the non-Markovian nature of fBm produces considerable obstacles to the well established analytic methods for the determination of the Flory index when we try to generalize them to fBm. As it turns out, ${ }^{4}$ numerical simulations are quite manageable. We give here some preliminary results for the case $d=1$, where the above-mentioned proposal would read

$$
v_{H}=\frac{2 H+2}{3}
$$

for $H \leq 1 / 2$ and $v_{H}=1$ otherwise. Numerically the authors found

$\begin{array}{ccc}H & v_{H} & \text { Simulation } \\ 0.10 & 0.73 & 0.772 \pm 0.037 \\ 0.20 & 0.80 & 0.803 \pm 0.012 \\ 0.35 & 0.90 & 0.917 \pm 0.013\end{array}$

in preliminary computations; systematic and more detailed calculations are presently under way. ${ }^{4}$

For illustration we show characteristic examples of simulated (two-dimensional) conformations for $N=30$, and Hurst indices $H=0.1, H=0.5$ and $H=0.9$ in Figs. 4-6.

\subsection{A remark on polymers in narrow tubes}

Finally we add a short remark on polymers in "nano-tubes". Following de Gennes, ${ }^{7}$ we consider tubes which are wider than the monomer size $a$, but much narrower than the polymer length $N a$.

$$
a<D \ll N a .
$$

Hence individual blobs of the polymer chain are restricted to a size $R \lesssim D$ and will contain

$$
N_{b} \sim D^{1 / v}
$$

monomers. Dense packing of the blobs in the tube would imply a polymer length in the tube of

$$
R_{T} \approx D \frac{N}{N_{b}} \sim N D^{1-1 / v}
$$

For $v \approx v_{H}$ and $d=3$ we would then have elongation with an inverse power of the tube diameter $D$, depending on the Hurst parameter $H$ :

$$
R_{T} \sim N D^{-\frac{3-2 H}{2+2 H}} .
$$

In like style it is also not hard to extend de Gennes' derivation of a non-linear Hooke's law for the stretching of polymers. 
$* * *$

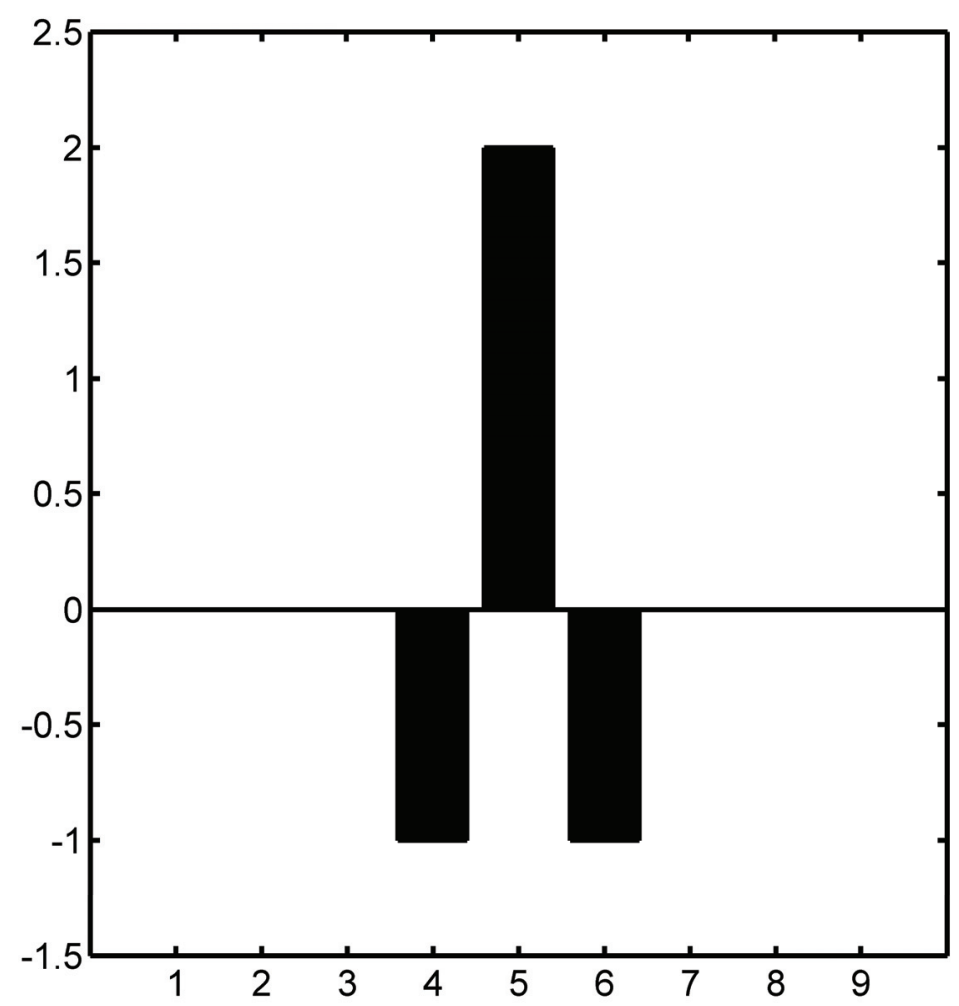

Figure $1 . \mathrm{H}=0.5$ 


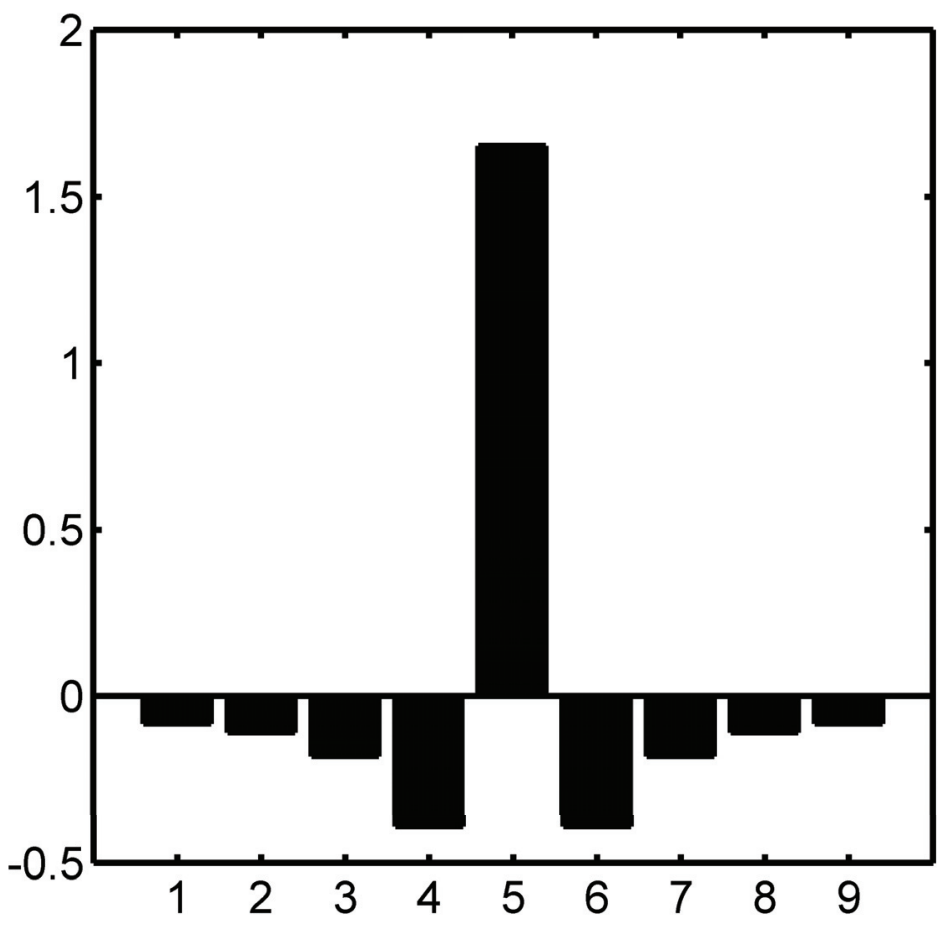

Figure 2. $\mathrm{H}=0.1$ 


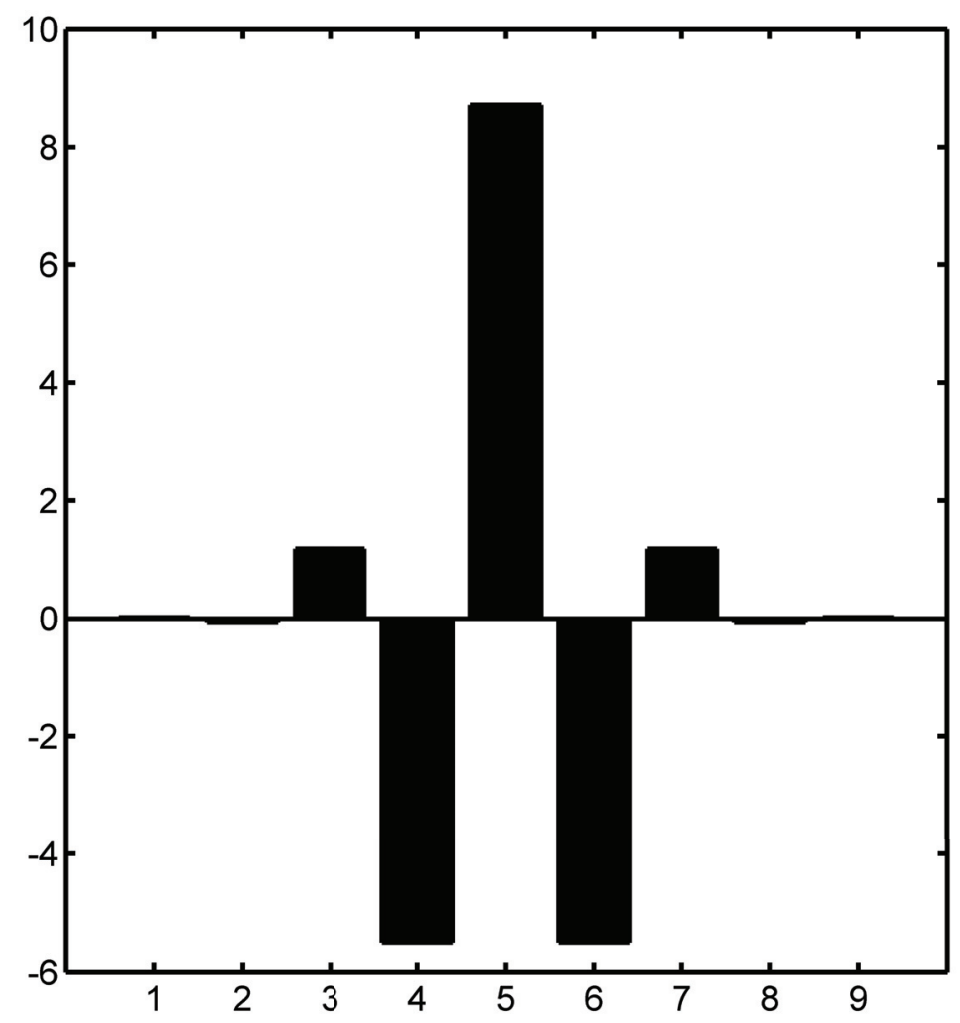

Figure $3 . \mathrm{H}=0.9$ 


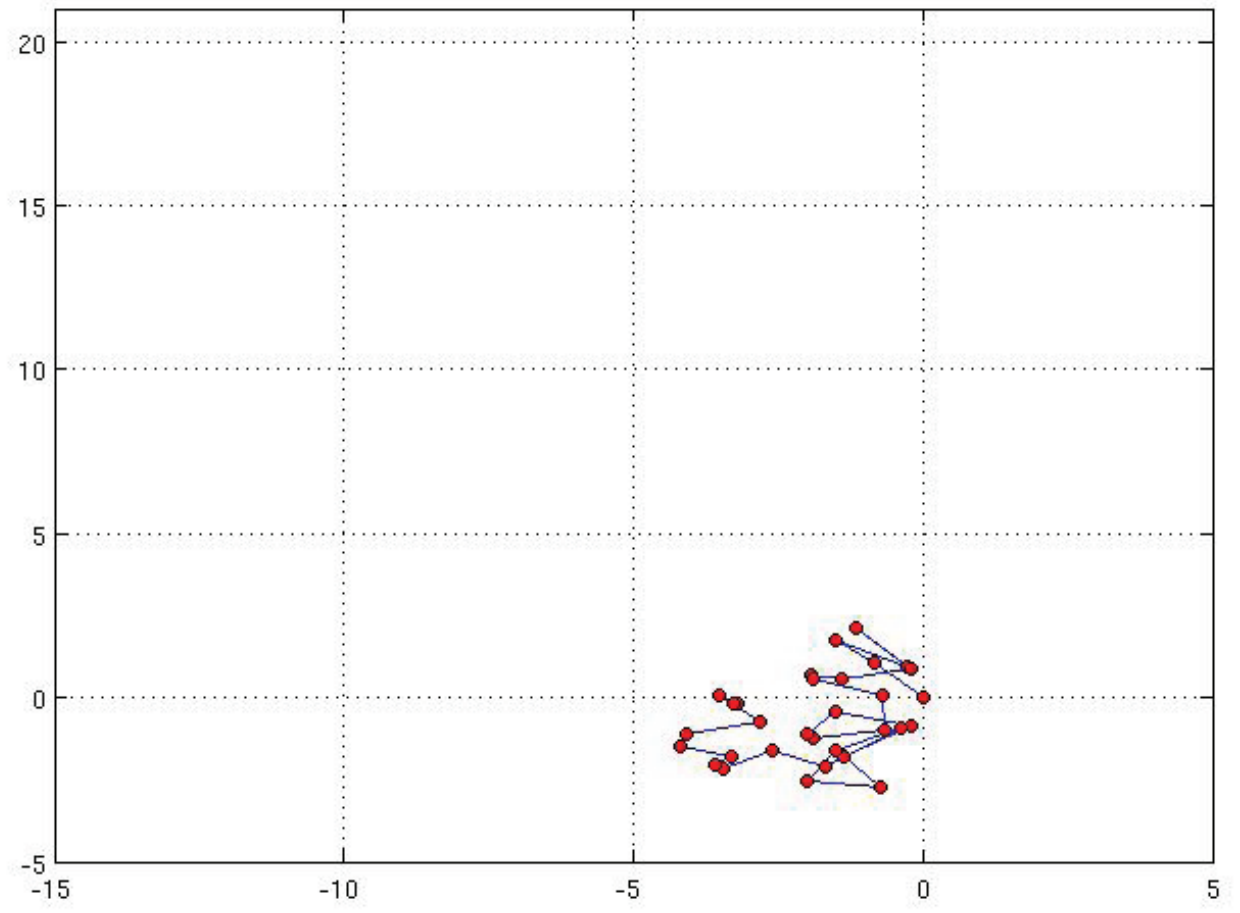

Figure 4. Self-repelling polymer: $\mathrm{H}=0.1$ 


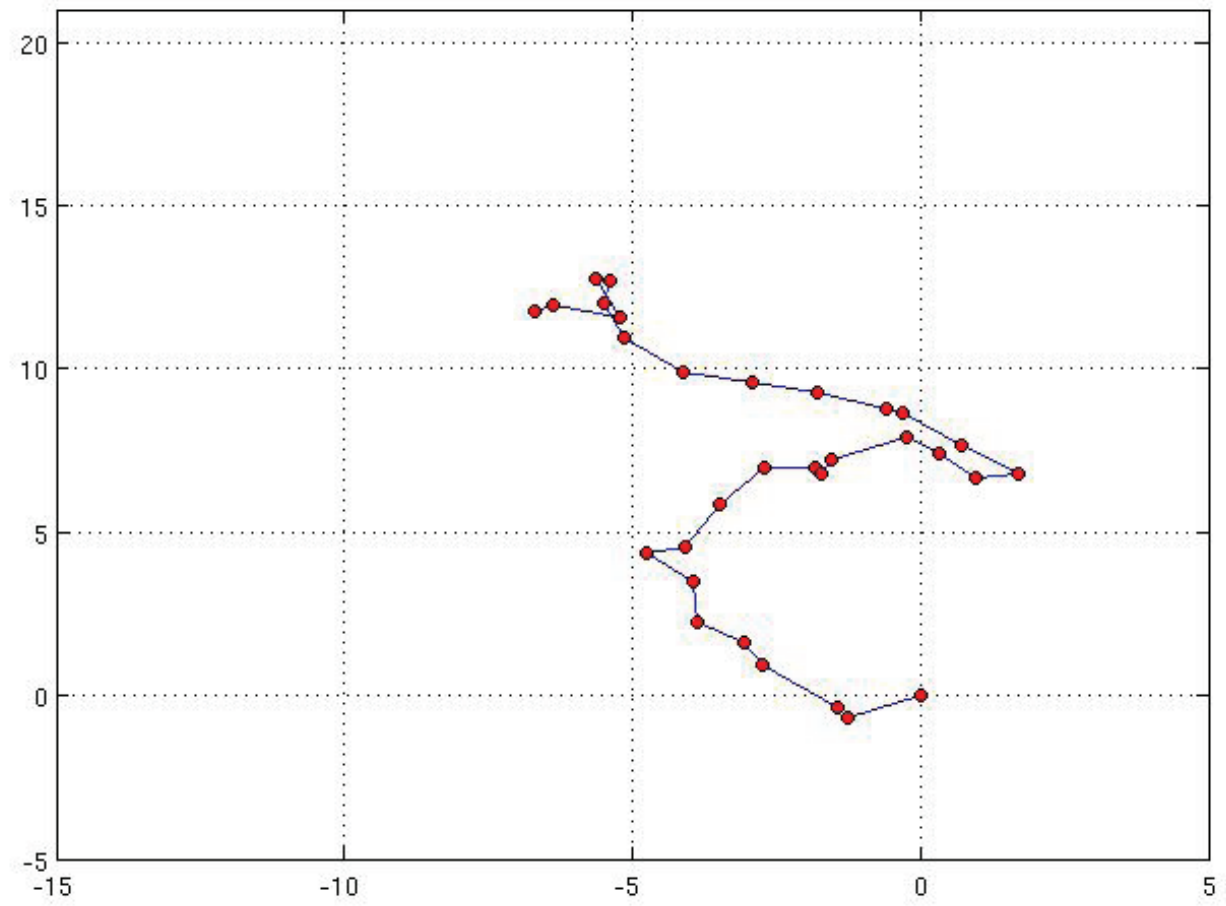

Figure 5. Self-repelling polymer: $\mathrm{H}=0.5$ 


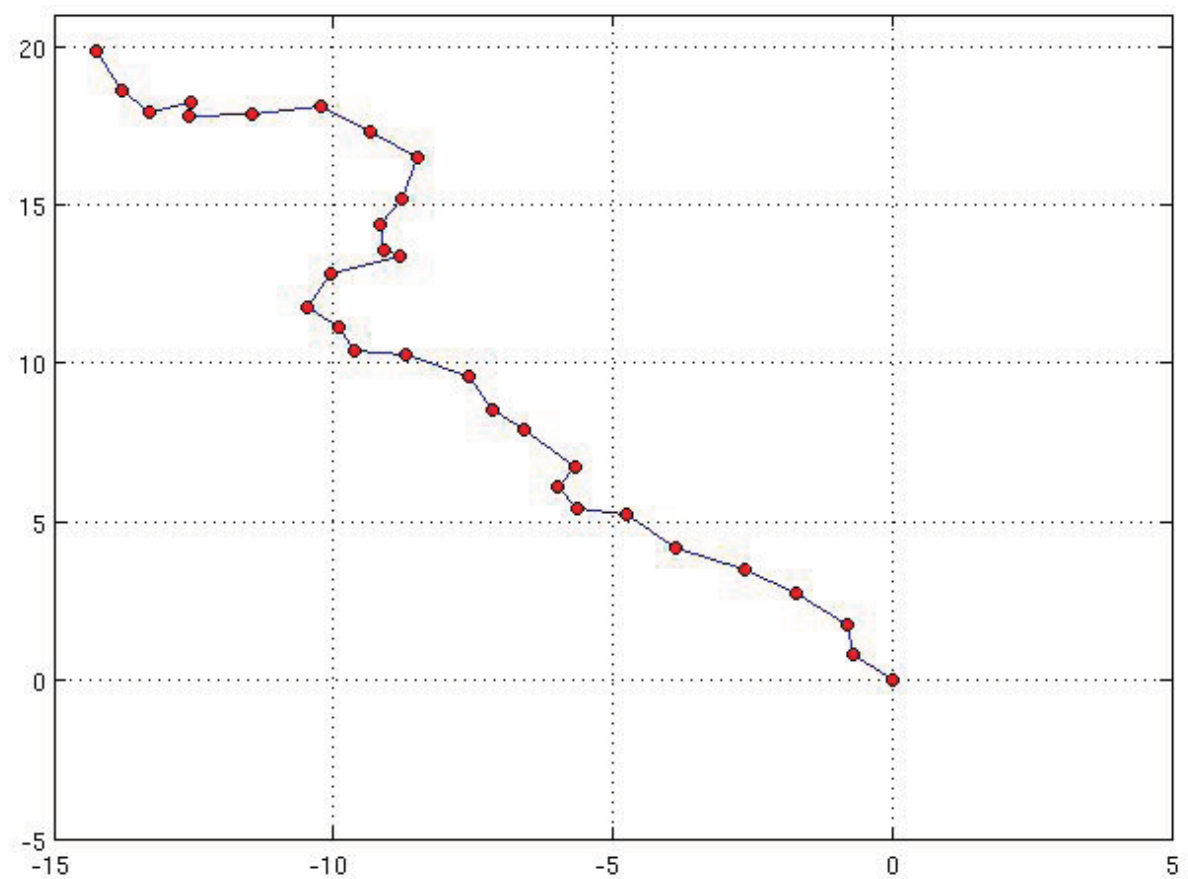

Figure 6. Self-repelling polymer: $\mathrm{H}=0.9$

\section{Acknowledgements}

We would like to express our gratitude for the splendid hospitality of our colleagues and friends Victoria Bernido and Christopher Bernido during a very pleasant stay at Jagna during the 6th Jagna International Workshop. We are happy to acknowledge helpful discussions with Chr. and M. C. Bernido, W. Bock, and M. Escobido. This work is supported by PEst OE/MAT/UI0209/2011, PEst-OE/MAT/UI0219/2011, PTDC/MAT/100983/2008 and a travel grant of the Alexander-v.-Humboldt Foundation.

\section{References}

1. G. Besold, H. Guo, M. J. Zuckermann: Off-lattice Monte Carlo simulation of the discrete Edwards model. J. Polymer Science B 38, 1053-1068 (2000).

2. F. Biagini, Y. Hu, B. Øksendal, T. Zhang: Stochastic Calculus for Fractional Brownian Motion and Applications. Springer, Berlin, 2007.

3. P. Biswas, B. J. Cherayil: Dynamics of Fractional Brownian Walks. J. Phys. Chem. 99, 816-821 (1995).

4. W. Bock et al.: Computer Studies of Fractional Brownian Polymers, in preparation. 
5. J. Bornales, M. J. Oliveira, L. Streit: Self-repelling fractional Brownian motion - a generalized Edwards model for chain polymers. To appear in Proceedings of ICQBIC 2011. World Scientific, Singapore. http://arxiv.org/abs/1106.3776

6. S. F. Edwards: The statistical mechanics of polymers with excluded volume. Proc. Roy. Soc. 85, 613-624 (1965).

7. P. G. de Gennes: Scaling Concepts in Polymer Physics. Cornell Univ. Press, Ithaca, NY, (1979).

8. M. Grothaus, M. J. Oliveira, J.-L. Silva, L. Streit: Self-avoiding fractional Brownian motion - The Edwards model. J. Stat. Phys. 145, 1513-1523 (2011).

9. R. van der Hofstad, W. König: A Survey of One-Dimensional Random Polymers. J. Stat. Physics, 103, 915-944 (2001).

10. Y. Hu and D. Nualart: Renormalized self-intersection local time for fractional Brownian motion. Ann. Probab., 33:948-983, 2005.

11. M. K. Kosmas, K. F. Freed: On scaling theories of polymer solutions. J. Chem. Phys. 69, 3647-3659 (1978).

12. Y. Mishura: Stochastic Calculus for Fractional Brownian Motion and Related Processes. Springer LNM 1929 (2008).

13. A. Pelissetto, E. Vicari: Critical phenomena and renormalization-group theory. Phys. Reports 368, 549-727 (2002).

14. M. Talagrand: Multiple points of trajectories of multiparameter fractional Brownian motion. Probab. Theory Related Fields 112, 545-563 (1998).

15. S. R. S. Varadhan: Appendix to "Euclidean Quantum Field Theory" by K. Symanzik, in: R. Jost, ed., Local Quantum Theory, Academic Press, New York, p. 285 (1969). 